\title{
Metodologia para o planejamento de aulas de hidroginástica
}

\author{
Metodology for planning shallow-water fitness
}

\author{
M.M. Olkoski, S.C. Matheus, E.C. Moraes, D. Tusset
}

ARTIGO ORIGINAL | ORIGINAL ARTICLE

\begin{abstract}
RESUMO
O objetivo desse estudo foi analisar uma metodologia para o planejamento de aulas de hidroginástica. Dezessete mulheres realizaram individualmente uma aula de hidroginástica. Obteve-se o consumo de oxigênio (analisador de gases TEEM 100) e a frequência cardíaca (frequencímetro Polar ${ }^{\circledR}$-Accurex Plus) a cada 20 segundos, sendo a concentração de lactato sanguíneo (lactímetro portátil Biosen 5030 EKFDiagnostic) e o índice de percepção de esforço (IPE) (Escala de Borg) verificados a cada 5 minutos e 50 segundos. A estatística utilizada apresentou valores médios e a variância (ANOVA OneWay com Post-hoc de Bonferroni) para cada variável entre as fases e os seis blocos da aula $(p<.05)$. Os resultados mostraram que as variáveis aumentaram progressivamente até o bloco 4 e partir do bloco 5 começaram a diminuir, confirmando a estrutura de pirâmide previamente elaborada. Os valores médios da fase principal da aula sugerem que a prática regular de aulas de hidroginástica nessas condições, provavelmente proporcionará a melhora do condicionamento físico aeróbico em sujeitos com características semelhantes aos do grupo avaliado, com IPE razoavelmente fácil. Conclui-se que a estrutura da aula sugerida poder ser eficiente na elaboração de aulas de hidroginástica.

Palavras-chave: variáveis fisiológicas, índice de percepção de esforço, hidroginástica
\end{abstract}

ABSTRACT

The aim to this study was analyze a methodology for a shallow-water fitness planning. Seventeen women performed individually shallow-water fitness. We obtained the oxygen uptake (gas analyzer TEEM 100) and heart rate (frequency meter Polar ${ }^{\circledR}$-plus Accurex) every 20 seconds. The blood lactate concentration (lactimeter portable Biosen 5030 EKF-Diagnostic) and rate perceived exertion (RPE) were collected every 5 minutes and 50 seconds. Mean, repeated-measures ANOVA and Bonferroni's post hoc tests were used for each variable between the phases and the six blocks of class $(p<.05)$. The variables increased progressively until block 4 and, from block 5 they started to decrease, which confirmed the pyramid structure suggested. The responses found at the main phase indicate that the physical fitness can be improved when shallow-water fitness is performed regularly and in the same situation as in this study, with a RPE fairly easy. In conclusion, this methodology structure seems to be efficient for others shallow-water fitness.

Keywords: physiological variables, rate perceived exertion, shallow-water fitness

Submetido: 21.05.2012 | Aceite: 15.05.2013

Mabel Micheline Olkoski. Universidade Estadual de Londrina, Brasil.

Silvana Corrêa Matheus, Eliane Corrêa de Moraes. Universidade Federal de Santa Maria, Brasil.

Dalila Tusset. Universidade de Brasília, Brasil.

Endereço para correspondência: Mabel Micheline Olkoski, Avenida Bento Gonçálves, n²174, Centro, CEP: 98465 000, Ametista do Sul, Rio Grande do Sul, Brasil.

E-mail: mabelolkoski@hotmail.com 
A hidroginástica é constituída de movimentos aquáticos específicos, que baseia-se no aproveitamento da resistência da água como sobrecarga (Kruel, Moraes, Ávila, \& Sampedro, 2001). No entanto, o planejamento dessas aulas deve ser realizado com cautela, levando-se em consideração as alterações fisiológicas proporcionadas pelo meio aquático (Alberton, Coertjens, Figueiredo, \& Kruel, 2005; Kruel et al., 2001). Sabe-se que em hidroginástica, os diferentes movimentos influenciam na intensidade de esforço onde o grupo muscular envolvido é proporcional à intensidade imposta (Alberton, Olkoski, Pinto, Becker, \& Kruel, 2007; Moraes, Kruel, Sampedro, \& Lopes, 2002). Além disso, o tempo de execussão dos diferentes exercícios de hidroginástica devem ser de 2 minutos e 20 segundos, para que as variáveis fisiológicas entrem em steady state (Moraes et al., 2002). Os estudos que analisaram aulas de hidroginástica (Alves, Mota, Costa, Cunha, \& Alves, 2004; Eckerson and Anderson, 1992; Gubiani, Neto, Petroski, \& Lopes, 2001; Kruel, Posser, Alberton, Pinto, \& Oliveira, 2009; Olkoski, Tosset, Wentz, \& Matheus, 2010; Tsourlou, Benik, Dipla, Zafeiridis, \& Kellis, 2006; Vickery, Cureton, \& Langstaff, 1983) não são consistentes quanto à justificativa da metodologia utilizada. Apenas os trabalhos de Olkoski et al. (2010) e de Kruel et al. (2009) tiveram cuidado em relação ao tempo e ao tipo de exercício durante a estruturação da aula, mas são questionados em relação à eficiência da estrutura metodológica (Olkoski et al., 2010) ou em função da melhora do condicionamento físico (Kruel et al., 2009).

Nesse contexto, verifica-se que os estudos não sustentam uma metodologia de planejamento de aulas de hidroginástica que além de proporcionar a melhora da resistência aeróbica, também possa disponibilizar a estruturação de aulas com diferentes exercícios mantendo-se uma estrutura pré-determinada e reconhecida na sua eficiência. Com isso, seria possível evitar a monotonia das aulas ao mesmo tempo em que se adequaria a diferentes populações. Além disso, a definição de uma metodologia tornará possível que outros estudos enfocando diferentes variáveis possam ser comparados fornecendo assim, maiores subsídios para os profissionais da área. Dessa forma, o presente estudo tem por objetivo analisar a eficácia de uma metodologia específica para o planejamento de aulas de hidroginástica.

\section{MÉTODO}

\section{Amostra}

Constituíram a amostra 17 mulheres (Tabela 1) voluntárias, universitárias, praticantes de hidroginástica há pelo menos seis meses e que não tomavam medicação que pudesse influenciar os resultados. As voluntárias foram informadas dos propósitos e procedimentos da pesquisa e assinaram o Termo de Consentimento Livre e Esclarecido aprovado pelo Comitê de Ética em Pesquisa da Universidade Federal de Santa Maria - UFSM/Brasil (0070.0.243.000-06).

\section{Tabela 1}

Valores médios (média) e desvio padrão (DP) apresentados pela amostra com relação à idade, massa corporal (MC), estatura e percentual de gordura corporal (GC\%)

\begin{tabular}{ccc}
\hline Variáveis & Média & DP \\
\hline Idade (anos) & 23.06 & 2.05 \\
MC (kg) & 54.29 & 6.62 \\
Estatura & 159.60 & 6.58 \\
GC (\%) & 22.73 & 3.88 \\
\hline
\end{tabular}

$\mathrm{Kg}=$ quilogramas.

\section{Instrumentos}

Para a obtenção da estatura (estadiômetro de madeira, resolução $0.5 \mathrm{~cm}$ ) o sujeito permaneceu em pé e com o corpo o mais ereto possível, mantendo o olhar fixo em um ponto a sua frente. A massa corporal (balança Welmy, resolução $0.100 \mathrm{~kg}$ ) foi verificada estando o sujeito em pé e de costas para a escala da balança.

As dobras cutâneas (compasso Cescorf ${ }^{T M}$, resolução $0.1 \mathrm{~mm}$ ) foram obtidas no hemicorpo 
direito e tomadas em triplicata, sendo a média utilizada para verificação da densidade corporal (Petroski, Pires, \& Simões, 1995) e posteriormente o percentual de gordura corporal (\%GC) através da fórmula de Siri (1961).

Foi verificado o consumo de oxigênio $\left(\mathrm{VO}_{2}\right)$ através do analisador de gases TEEM 100 AeroSport Inc. e a frequência cardíaca (FC) através do frequêncímetro da marca Polar ${ }^{\circledR}$-Accurex Plus.

Também foram obtidas amostras de sangue venoso, coletadas em um capilar EKF-Diagnostic, preenchido completamente e colocado em um micro tubo do tipo eppendorf (EKF-Diagnostic). Após, as amostras foram analisadas pelo lactímetro portátil Biosen 5030 (EKF-Diagnostic) para a obtenção da concentração de lactato sanguíneo [Lac].

A escala de Borg (2000) foi utilizada para verificação do índice de percepção de esforço (IPE) indicado pelo sujeito que realizou o exercício e para reprodução das músicas durante todas as aulas, foi utilizado um aparelho de som compact disc da marca Toshiba.

A aula de hidroginástica foi realizada em um tanque de pesagem hidrostática. Essa estrutura tem $1.71 \mathrm{~m}$ de diâmetro, $1.50 \mathrm{~m}$ de profundidade o que torna possível controlar a temperatura da água (termômetro de mercúrio, da marca Incoterm $^{\circledR}$ ) e a profundidade de imersão (através da evasão ou colocação de água).

\section{Procedimentos}

Todas as voluntárias foram avaliadas na fase pós-menstrual $\left(6^{\circ}-10^{\circ}\right.$ dia). Para tanto, elas compareceram em data e horário previamente estabelecido no Laboratório de Cineantropometria (Labcine) da UFSM/Brasil para serem inicialmente submetidas às avaliações antropométricas. Seguiu-se, enquanto permaneceram sentadas, a assepsia no lóbulo da orelha e colocou-se uma fina camada da pomada vasodilatadora Finalgon. Passados 20 minutos, retirou-se a pomada e perfurou-se o local com uma micro-lanceta descartável, coletando-se o sangue capilar. Posteriormente, a voluntária foi monitorada pelo analisador de gases $\left(\mathrm{VO}_{2}\right)$ e pelo frequencímetro (FC), permanecendo mais 5 minutos sentada. Os valores de $\mathrm{FC}$, de $\mathrm{VO}_{2}$ e da [Lac] verificados nesses momentos, foram considerados de repouso.

$\mathrm{Na}$ sequência, a voluntária realizou individualmente a aula de hidroginástica. A temperatura da água foi mantida entre 32 e $33^{\circ} \mathrm{C}$, considerada termoneutra para exercícios no meio líquido (McArdle, Magel, Lesmes, \& Pechar, 1976). A profundidade de imersão foi na altura do processo xifóide (Alberton et al., 2007; Olkoski et al., 2010) e utilizou-se a mesma sequência de músicas (136bpm) para todos os sujeitos.

A estrutura de aula da presente metodologia foi composta por três fases: inicial, principal e final. $\mathrm{Na}$ fase inicial (5 minutos) foram realizados alongamentos (deltóide, peitorais, dorsais, quadríceps e panturrilha) e aquecimentos articulares (cervical, ombros, cotovelos, punhos, quadril, joelho e tornozelo). Na fase principal (35 minutos), foram realizados exercícios aeróbicos de movimentos de membros superiores (MMS) realizados concomitantemente com os membros inferiores (MMI), totalizando 30 exercícios. Esta fase foi composta por 6 blocos, determinados pelos MMI, pois neles encontram-se os maiores grupos musculares (Alberton et al., 2007). O mesmo MMI foi mantido durante 5 minutos e 50 segundos. $\mathrm{O}$ primeiro e o último bloco foram caracterizados por MMI que envolviam pequenos grupos musculares. Já nos blocos 2 e 5 foram realizados MMI com maiores grupos musculares. Durante os blocos 3 e 4 os MMI foram compostos por grandes grupamentos musculares e por uma grande área projetada, também importante na determinação da intensidade de esforço (Alberton et al., 2007; Pöyhönen, Keskinen, Hautala, \& Mälkiä, 2000). Os MMS foram alterados a cada 1 minuto e 10 segundos, sendo que o mesmo grupo muscular foi mantido por 2 minutos e 20 segundos (Moraes et al., 2002), fazendo com que para cada MMI fossem utili- 
zados cinco MMS. No total foram 6 MMI e 30 MMS, formando os seis blocos de exercícios durante a fase principal da aula. A metodologia sugerida para a fase principal pode ser visualizada na tabela número 2 :

Tabela 2

Parte principal da aula de hidroginásitca e seus respectivos exercícios e tempos de execussão

\begin{tabular}{|c|c|c|c|c|c|}
\hline Bloco & MMI & $\begin{array}{c}\text { Tempo } \\
\text { do MMI }\end{array}$ & MMS & $\begin{array}{c}\text { Tempo } \\
\text { do MMS }\end{array}$ & $\begin{array}{c}\text { Característica } \\
\text { S do MMI }\end{array}$ \\
\hline 1 & $\begin{array}{l}1 \text { - Extensão de joelho } \\
\text { até } 45^{\circ}\end{array}$ & $5 ’ 50 \prime$ & $\begin{array}{l}1 \text { - Extensão de braços alternados no plano } \\
\text { frontal; } \\
2 \text { - Flexão/extensão de cotovelos no plano } \\
\text { sagital; } \\
3 \text { - Adução/abdução frontal de ombros; } \\
4 \text { - Adução/abdução posterior de ombros; } \\
5 \text { - Extensão de braços no plano frontal. }\end{array}$ & $\begin{array}{l}\text { 1'10" } \\
1 ' 10 ”\end{array}$ & $\begin{array}{l}\text { Pequenos } \\
\text { grupos } \\
\text { musculares }\end{array}$ \\
\hline 2 & 2 - Flexão do quadril & $50^{\prime \prime}$ & $\begin{array}{c}1 \text { - Extensão de braços na frontal e posterior; } \\
2 \text { - Adução de ombros alternado ao joelho } \\
\text { oposto; } \\
3 \text { - Movimento da braçada do nado peito; } \\
4 \text { - Extensão de braços alternados a perna para } \\
\text { baixo; } \\
5 \text { - Flexão/extensão horizontal e alternado de } \\
\text { cotovelos }\end{array}$ & 1'10”' & $\begin{array}{l}\text { Grandes grupos } \\
\text { musculares }\end{array}$ \\
\hline 3 & $\begin{array}{l}3 \text { - Chute a frente/ } \\
\text { flexão do quadril }\end{array}$ & 5’50”' & $\begin{array}{c}1 \text { - Flexão/extensão horizontal de ombros e } \\
\text { adução/abdução de ombros; } \\
2 \text { - Adução das escápulas; } \\
3 \text { - Extensão de braços alternados a perna no } \\
\text { plano frontal; } \\
4 \text { - Extensão/flexão de cotovelos na vertical; } \\
5 \text { - Extensão/flexão de cotovelos na horizontal. }\end{array}$ & $\begin{array}{l}\text { 1'10" } \\
\text { 1'10" } \\
\text { 1'10" } \\
\text { 1'10" }\end{array}$ & $\begin{array}{l}\text { Grandes grupos } \\
\text { musculares } \\
\text { e áreas } \\
\text { projetadas }\end{array}$ \\
\hline 4 & $\begin{array}{c}4 \text { - Flexão do quadril } \\
\text { em abdução/perna } \\
180^{\circ}\end{array}$ & $5 ’ 50 \prime$ & $\begin{array}{c}1 \text { - Movimentos de abraçar a água; } \\
2 \text { - Flexão/extensão horizontal de ombros com } \\
\text { cotovelos flexionados em } 90^{\circ} \text {; } \\
3 \text { - Extensão de braços no plano frontal e ao } \\
\text { lado; } \\
4 \text { - Extensão alternada de braços contrários à } \\
\text { perna; } \\
5 \text { - Abdução/adução de ombros no plano } \\
\text { posterior }\end{array}$ & 1'10”' & $\begin{array}{l}\text { Grandes grupos } \\
\text { musculares } \\
\text { e áreas } \\
\text { projetadas }\end{array}$ \\
\hline 5 & 5 - Flexão de joelhos & $5 ’ 50 \prime$ & $\begin{array}{c}1 \text { - Abdução/adução de ombros no plano } \\
\text { posterior alternadamente; } \\
2 \text { - Extensão de braços frontal; } \\
3 \text { - Flexão de cotovelo na vertical; } \\
4 \text { - Flexão de ombros; } \\
5 \text { - Adução/abdução de ombros. }\end{array}$ & $\begin{array}{l}\text { 1'10" } \\
\text { 1'10"' } \\
\text { 1'10"' } \\
\text { 1'10"' } \\
\text { 1'10"' }\end{array}$ & $\begin{array}{l}\text { Grandes grupos } \\
\text { musculares }\end{array}$ \\
\hline 6 & 6 - Deslize frontal & $5 ’ 50 \prime$ & $\begin{array}{l}1 \text { - Extensão alternada de braços no plano } \\
\text { frontal } \\
2 \text { - Flexão/extensão de cotovelos na horizontal; } \\
3 \text { - Flexão/extensão de ombros; } \\
4 \text { - Flexão/extensão horizontal de ombros; } \\
5 \text { - Extensão de braços na vertical e no plano } \\
\text { frontal. }\end{array}$ & $\begin{array}{l}\text { 1'10"' } \\
\text { 1'10"' }\end{array}$ & $\begin{array}{l}\text { Pequenos } \\
\text { grupos } \\
\text { musculares }\end{array}$ \\
\hline
\end{tabular}


Cabe ressaltar, que todos os exercícios aqui sugeridos podem ser alterados em outras aulas, uma vez que os tempos de MMI e MMS e a Característica do MMI sejam mantidos. Nesse sentido, espera-se que as variáveis estudadas aumentem progressivamente até os blocos 3 e 4 para então começarem a diminuir, respeitando uma estrutura de pirâmide (Dantas, 1998).

A fase final teve duração de 5 minutos, onde foram realizados alongamentos (deltóide, bíceps, tríceps, peitorais, dorsais, quadríceps, isquiotibiais e panturrilha).

Além das coletas de repouso, a [Lac] também foi obtida ao final da fase inicial, de cada bloco da fase principal e logo após a fase final. Os dados de $\mathrm{VO}_{2}$ e de $\mathrm{FC}$ foram obtidos a cada 20 segundos durante toda a aula, sendo que a média dos últimos 3 minutos de cada bloco e cada fase foi utilizada para posterior análise. Para a obtenção dos dados de IPE, a Escala de Borg (2000) foi apresentada à avaliada 30 segundos antes do final de cada fase e de cada bloco da fase principal.

\section{Análise Estatística}

Para analisar os dados coletados foram utilizados valores médios de $\mathrm{FC}$ e do $\mathrm{VO}_{2}$. Testou-se a normalidade (Shapiro-Wilk), a homogeneidade (Levene) e a variância (ANOVA OneWay) de cada variável entre as fases e os seis blocos da aula, sendo que através do Post hoc de Bonferroni foi possível verificar as diferenças encontradas. O nível de significância adotado foi de $5 \%$ e o pacote estatístico utilizado foi o SPSS $v s$ 13.0.

\section{RESULTADOS}

A tabela 3 mostra a variação dos valores médios observados durante as fases e os seis blocos da aula. De forma geral, pode-se verificar que as variáveis aumentam progressivamente até o bloco 4 e partir do bloco 5 começaram a diminuir.

Foram obtidos valores médios das variáveis estudadas durante a fase principal da aula. Esses resultados podem ser observados na tabela 4. Os resultados da [Lac] permaneceram entre 2 e $4 \mathrm{mmol}$ e o IPE médio foi razoavelmente fácil.

Tabela 4

Valores médios (média) e desvio padrão (DP) da [Lac], do $V_{2}$, da FC e do IPE na fase principal da aula

\begin{tabular}{lll} 
Variáveis & Média & DP \\
\hline$[\mathrm{Lac}](\mathrm{mmol})$ & 3.58 & 1.69 \\
$\mathrm{VO}_{2}(\mathrm{ml} / \mathrm{kg} \cdot \mathrm{min})$ & 23.22 & 3.77 \\
$\mathrm{FC}(\mathrm{bpm})$ & 146.65 & 14.71 \\
$\mathrm{IPE}$ & 11 & 2 \\
\hline
\end{tabular}

[Lac] = Concentração de lactato sanguíneo; $\mathrm{VO}_{2}=$ Consumo de oxigênio; $\mathrm{FC}$ = Frequência cardíaca; IPE = Índice de percepção de esforço.

\section{DISCUSSÃO}

Os resultados das diferentes fases da aula mostram que a estrutura aqui sugerida está dentro do esperado. Visto que, em um primeiro momento os valores encontrados na fase inicial estão abaixo do recomendado para exercício

Tabela 3

Análise de variância dos valores médios entre as 3 fases e os 6 blocos da aula de hidroginástica

\begin{tabular}{|c|c|c|c|c|c|c|c|c|}
\hline \multirow{2}{*}{ Variáveis } & \multirow{2}{*}{ Fase Inicial } & \multicolumn{6}{|c|}{ Fase Principal (Blocos) } & \multirow{2}{*}{ Fase Final } \\
\hline & & 1 & 2 & 3 & 4 & 5 & 6 & \\
\hline [Lac] $(\mathrm{mmol})$ & $1.58 \mathrm{~b}$ & $2.50 \mathrm{~cd}$ & $2.57 c d$ & $4.26 \mathrm{ad}$ & $4.46 a$ & 4.23ad & $3.42 \mathrm{~d}$ & $2.89 \mathrm{~cd}$ \\
\hline VO2 (ml/kg.min) & $7.08 \mathrm{e}$ & $16.59 \mathrm{~d}$ & $20.58 d$ & $28.20 \mathrm{a}$ & $29.55 \mathrm{a}$ & $24.30 \mathrm{~d}$ & $20.07 d$ & $7.10 \mathrm{e}$ \\
\hline FC (bpm) & $91.58 \mathrm{~d}$ & $123.12 c$ & $136.15 c$ & $158.34 \mathrm{ba}$ & $163.38 \mathrm{a}$ & $154.59 \mathrm{ab}$ & $144.41 b c$ & $112.24 \mathrm{e}$ \\
\hline IPE & $7 a$ & $8 a$ & $10 \mathrm{~b}$ & $12 c$ & $13 c$ & $12 c$ & $11 b c$ & $7 a$ \\
\hline
\end{tabular}

Letras diferentes para a mesma variável apresentam diferenças estatisticamente significativa $(p<.05)$ entre as fases e os 6 blocos. [Lac] = Concentração de lactato sanguíneo; $\mathrm{VO}_{2}=$ Consumo de oxigênio; $\mathrm{FC}=$ Frequência cardíaca; IPE = Índice de percepção de esforço. 
aeróbico, atingindo o objetivo de aquecimento já que aumentaram significativamente na fase principal onde a [Lac] mostrou que o trabalho aeróbico foi predominante (Kindermann, 1979). No último bloco da fase principal para a fase final, apenas a [Lac] não diminuiu para retornar aos valores de repouso. Moraes et al. (2002) afirmam que após exercícios de hidroginástica, a [Lac] leva em média 30 minutos para voltar as situações de repouso, justificando esses valores encontrados.

Em um segundo momento, a eficiência da metodologia é verificada ao direcionarmos um olhar específico para a fase principal da aula. Como esperado, as variáveis aumentam progressivamente a cada bloco até o 4 e a partir de então diminuem, impondo ao organismo diferentes esforços no decorrer da aula. Apesar da diferença de esforço não ter sido significativa em todos os blocos nem para todas as variáveis, esses resultados mostram que o tipo de movimento (Alberton et al., 2007; Pöyhönen et al., 2000) e o tempo em que deve ser mantido (Moraes et al., 2002) estão corretamente estruturados no método aqui sugerido, já que a aula realmente demonstrou ter uma estrutura de pirâmide.

Assim, o tempo de execução de cada MMI e MMS e a Característica do MMI podem ser considerados aqui como a base metodológica. Sugere-se que durante o dia-a-dia de aula, os profissionais alterem os exercícios de hidroginástica e mantenham a metodologia (em relação ao tempo e a característica do exercício). Com isso, espera-se que aulas de hidroginástica sejam dinâmicas e eficientes.

Outros estudos com aulas de hidroginástica já foram realizados. No entanto, eles não respeitam o tempo de movimento (Vickery et al., 1983) ou então não justificam de forma adequada a metodologia utilizada (Olkoski et al., 2010). Em outro trabalho (Kruel et al., 2009), essas duas questões foram respeitadas mas os autores não informam a eficiência da aula para a melhora do condicionamento físico, considerado importante enquanto exercício.

No presente estudo, o tempo e a característica do movimento foram respeitados e além dessa estrutura de aula ter sido confirmada (Tabela 3), os resultados médios de [Lac] verificados na fase principal da aula (Tabela 4) mostram que os sujeitos realizaram a aula dentro da faixa indicada para a melhora do condicionamento cardiorrespiratório (Kinderman, 1979).

Em outro trabalho, Olkoski et al. (2010) confirmaram a eficiência dessa metodologia de aula na melhora do condicionamento cardiorrespiratório avaliada através da $\mathrm{FC}$ e do $\mathrm{VO}_{2}$. Esses estudiosos constataram que a intensidade de esforço também em termos percentuais estava dentro dos padrões estabelecidos pela literatura (ACSM, 2003) para exercícios aeróbicos.

No trabalho de Kruel et al. (2009), foi estudado o método contínuo (IPE de 13) e método intervalado (IPE de 9 e 17). As aulas foram realizadas em situações e por um grupo semelhante ao do presente estudo. Verificou-se que a aula a partir do método intervalado foi a que impôs maior intensidade, sendo considereada pelos referidos autores como a mais efetiva na melhora do condiconamento fisico. Cabe ressaltar que os valores médios da $\mathrm{FC}$ e do $\mathrm{VO}_{2}$ respectivamente à aula intervalada (132.80 $\pm 15.70 \mathrm{bpm}$ e $\left.15.50 \pm 2.80 \mathrm{ml} / \mathrm{kg} \cdot \mathrm{min}^{-1}\right)$ e à aula contínua $(118.00 \pm 14.80 \mathrm{bpm}$ e 10.70 $\pm 2.70 \mathrm{ml} / \mathrm{kg} \cdot \mathrm{min}^{-1}$ ) são inferiores aos encontrados no presente trabalho (Tabela 4). Assim, questiona-se se essa aula seria mesmo eficiente na melhora do condicionamento físico. Além disso, ressalta-se que esses estudiosos controlaram a intensidade dessas aulas através do IPE. Graef e Kruel (2006) afirmam que não há consenso sobre a correlação dessa variável com a $\mathrm{FC}$ e com o $\mathrm{VO}_{2}$ durante exercícios no meio líquido e que talvez a prescrição do treino a partir do IPE não reflita realmente alterações orgânicas eficientes para a melhora do condicionamento físico. 
No presente estudo, a aula não foi prescrita através de uma variável de controle, mas a estrutura do método sugerido parece impor uma intensidade de esforço maior do que a encontrada no trabalho de Kruel et al. (2009) e com a prática regular, provavelmente seja eficaz na melhora do condicionamento físico, visto que as respostas fisiológicas aqui encontradas sustentariam resultados positivos com a sua prática (ACSM, 2003). Além disso, a praticidade de uma situação onde apenas a estrutura da aula oferece a intensidade adequada para o aluno impõe uma maior praticidade no dia-a-dia de aula.

Outro dado interessante obtido no presente trabalho é a sensação de esforço do sujeito (IPE) frente ao exercício realizado. Durante a fase principal da aula o IPE médio foi 11, correspondendo a um esforço razoavelmente fácil (Borg, 2000). Isso significa dizer que apesar do esforço físico imposto aos sujeitos estarem dentro dos padrões indicados (verificado pelas variáveis fisiológicas), o esforço percebido pelo grupo foi ameno, ou seja, a aula de hidroginástica aqui estudada parece proporcionar uma melhora do condicionamento aeróbico ao mesmo tempo em que oferece bem-estar durante o exercício.

\section{CONCLUSÕES}

Conclui-se que a metodologia aqui sugerida é eficaz, confirmada através de análises fisiológicas. Além disso, a prática regular de aulas de hidroginástica nessas condições, provavelmente proporcionará a melhora do condicionamento físico em sujeitos com características semelhantes às do grupo estudado, com uma percepção de esforço razoavelmente fácil.

\section{Agradecimentos:}

Nada declarado.

\section{Conflito de Interesses:}

Nada declarado.
Financiamento:

Nada declarado.

\section{REFERÊNCIAS}

Alberton, C.L., Coertjens, M., Figueiredo, P.A., \& Kruel, L.F. (2005). Behavior of oxygen uptake in water exercise performed at different cadences in and out of water. Medicine and Science in Sports and Exercise, 37:S103.

Alberton, C.L., Olkoski, M.M., Pinto, S.S., Becker, M.E., \& Kruel, L.F.M. (2007). Cardiorrespiratory responses of postmenopausal women to different water exercises. International Journal of Aquatic Research and Education, 4(1),363-372.

Alves, R.V., Mota, J., Costa, M.C., \& Alves, J.G.B. (2004). Aptidão física relacionada à saúde de idosos: influência da hidroginástica. Revista Brasileira de Medicina do Esporte, 10(1), 31-37.

American College of Sports Medicine (ACSM). (2003). Manual de Pesquisa das Diretrizes do ACSM para Testes de Esforço e sua Prescrição. Rio de Janeiro: Guanabara Koogan.

Borg, G. (2000). Escalas de Borg para dor e o esforço percebido. São Paulo: Manole.

Dantas, E. (1998). A prática da preparação física. Rio de Janeiro: Shape.

Eckerson, J., \& Anderson, T. (1992). Physiological response to water aerobics. Journal of Sports Medicine and Physical Fitness, 32 (1),255-261.

Graef, F.I., \& Kruel, L.F.M. (2006). Frequência cardíaca e percepção subjetiva do esforço no meio aquático: diferenças em relação ao meio terrestre e aplicações na prescrição do exercício - uma revisão. Revista Brasileira de Medicina do Esporte, 12(4),221-228.

Gubiani, G.L., Neto, C.S.P., Petroski, É.L., \& Lopes, A.S. (2001). Efeitos da hidroginástica sobre indicadores antropométricos de mulheres entre 60 e 80 anos de idade. Revista Brasileira de Cineantropometria e Desempenho Humano, 3(1),34-41.

Kindermann, W. (1979). The significance of the aerobic-anaerobic transition for the determination of work load intensities during endurance training. European Journal of Applied Physiological, 
42(1),25-34.

Kruel, L.M.F., Moraes, E.Z.C., Ávila, A.O.V., \& Sampedro, R.M.F. (2001). Alterações fisiológicas e biomecânicas em indivíduos praticando exercícios de hidroginástica dentro e fora d'água. Kinesis, no especial, 104-154.

Kruel, L.F.M., Posser, M.S., Alberton, C.L., Pinto, S.S., \& Oliveira, A.S. (2009). Comparison of energy expenditure between continuous and interval water aerobic routines. International Journal of Aquatic Research and Education, 3(2), 186-196.

McArdle, W.D., Magel, J.R., Lesmes, G.R., \& Pechar, C.S. (1976). Metabolic and cardiovascular adjustment to work in air and water at 18,25 and $33^{\circ} \mathrm{C}$. Journal of Applied Physiology, 40(1),85-90.

Moraes, E.Z.C., Kruel, L.F.M., Sampedro, R.M.F., \& Lopes, L.F.D. (2002). Metodologia de medida de esforço para exercícios de hidroginástica em diferentes profundidades de água. Kinesis, 27(4),43-64.

Olkoski, M.M., Tosset, D., Wentz, M.D., \& Matheus, S.C. (2010). Comportamento de variáveis fisiológicas durante a aula de hidroginástica com mulheres. Revista Brasileira de Cineantropometria e Desempenho Humano, 12(1),43-48. doi:10.5007/1980-0037.2010v12n1p43
Petroski, E.L., Pires, N., \& Simões, C. (1995). Validação de equações antropométricas para a estimativa da densidade corporal em mulheres. Revista Brasileira de Atividade Física \& Saúde, 1(2),65-73.

Pöyhönen, T., Keskinen, K.L., Hautala, A., \& Mälkiä, E. (2000). Determination of hydrodinamic drag forces and drag coefficients on human leg/foot model during knee exercise. Clinical Biomechy, 15(4),256-260. doi: 10.1016/S02680033(99)00070-4

Siri, W.E. (1961). Body composition from fluid spaces and density: analysis of methods. In Brozek, J., \& Henschel, A. (Eds.), Thechniques for measuring body composition (pp. 223-224). Washington: National Academy of Sciences.

Tsourlou, T., Benik, A., Dipla, K., Zafeiridis, A., \& Kellis, S. (2006). The effects of a twenty-four-week aquatic training program on muscular strength performance in healthy elderly women. Journal of Strength and Conditioning Research, $4(1), 811-818$.

Vickery, S.R., Cureton, K.J., \& Langstaff, J.L. (1983). Heart rate and energy expenditure during aqua dynamics. Physical Sports and Medicine, 11 (3),67-73.

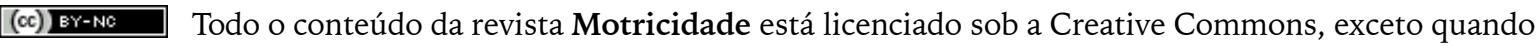
especificado em contrário e nos conteúdos retirados de outras fontes bibliográficas. 